\title{
Persistent COVID-19 Infection in Wiskott-Aldrich Syndrome Cleared Following Therapeutic Vaccination: a Case Report
}

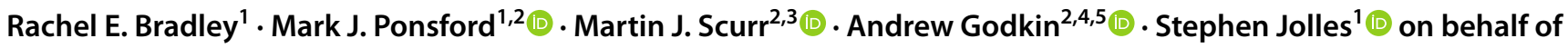 \\ On behalf of the Immunodeficiency Centre for Wales
}

Received: 15 September 2021 / Accepted: 13 October 2021 / Published online: 29 October 2021

(c) The Author(s) 2021

\section{To the Editor}

Interventions to address viral persistence of COVID19 in immunosuppressed individuals are urgently needed for both patient and societal benefit [1]. Infection with the novel pandemic coronavirus, SARS-CoV-2, has recently been reported up to 315 days in adults with hematological malignancies [2, 3]. These cases demonstrated recovery of infectious "live" virions and accumulation of mutations favoring vaccine escape during protracted SARS-CoV-2 infection [2, 3]. Immunodeficient individuals have thus been suggested as a reservoir for endemicity and a potential threat to the global vaccination effort. This mirrors wider experience with primary immunodeficiency, where excretion of neuro-virulent vaccine-derived polio virus has been described in association for up to 28 years [4]. To date, reports of protracted SARS-CoV-2 infections have focused on symptomatic cases, with varying therapeutic success [2, $3,5]$. The broad spectrum of SARS-CoV-2 presentations and frequently atypical nature of disease in immunosuppressed individuals led us to suspect viral persistence may also occur in pauci-symptomatic cases. Here we describe a case of protracted SARS-CoV-2 infection for 218 days in a patient

Rachel E. Bradley and Mark J. Ponsford contributed equally as joint first authors.

Stephen Jolles

jollessr@cardiff.ac.uk

1 Immunodeficiency Centre for Wales, University Hospital of Wales, Cardiff, UK

2 Division of Infection \& Immunity, School of Medicine, Cardiff University, Cardiff, UK

3 ImmunoServ LTD, Cardiff, UK

4 Systems Immunity University Research Institute, School of Medicine, Cardiff University, Cardiff, UK

5 Department of Gastroenterology \& Hepatology, University Hospital of Wales, Cardiff, UK with Wiskott-Aldrich syndrome (WAS), an inborn error of metabolism predisposing to dysregulated immunity and infection. We find that mRNA vaccination allowed induction of humoral and cellular immune responses to SARS-CoV-2, which had not been triggered by the ongoing infection itself, followed by viral clearance.

\section{Case Description}

A 37-year-old Caucasian male with Wiskott-Aldrich syndrome (WAS) (IVS6 + 5G>A) developed anosmia and ageusia, followed by cough, dyspnoea, and fatigue (henceforth day 0). Community nasopharyngeal reverse-transcription PCR (RT-PCR) testing 1 day later was positive for SARSCoV-2. Contact tracing suggested workplace exposure. Past medical history included childhood splenectomy for thrombocytopenia, eczema, recurrent infections, early bronchiectasis, asthma, and persistent molluscum contagiosum. Following diagnosis of specific antibody deficiency aged 24 years (based on suboptimal conjugated and unconjugated vaccine responses and reduced class switched memory $B$ cells, described online supplementary), he was commenced on subcutaneous immunoglobulin replacement (SCIg) and maintained on $8 \mathrm{~g} /$ week with trough $\mathrm{IgG}$ levels of $12-15$ $\mathrm{g} / \mathrm{L}$. Sinopulmonary infection history included RT-PCR-confirmed HKU1-coronavirus infection 12 months previously.

The patient was monitored via our virtual COVID-19 ward. Home pulse oximetry showed maintained peripheral saturations $>96 \%$ on room air and admission to hospital was not indicated. Incomplete improvement in sense of smell and taste was noted after 2 months; however, he experienced fluctuating symptoms of chest tightness, dyspnoea, poor concentration, and fatigue approximately every fourth week and serial RT-PCR testing remained persistently positive (Fig. 1A, B). Inflammatory markers such as C-reactive protein (CRP) remained less than $12 \mathrm{mg} / \mathrm{L}$ and 
Fig. 1 Viral load and immune response relative to vaccination. Semi-quantitative reverse transcriptase polymerase chain reaction (RT-PCR, Perkin-Elmer) detection of SARS-CoV-2 by A N-gene and B ORF1a-gene. C: interferon-gamma $\mathrm{T}$ cell responses to both SARS-CoV-2 nucleocapsid and spike peptide pool stimulation. D Anti-SARSCoV-2 spike $\mathrm{S} 1$-domain IgG serum response. Vertical dashed lines indicate timing of 1 st and 2 nd doses of mRNA vaccination
A

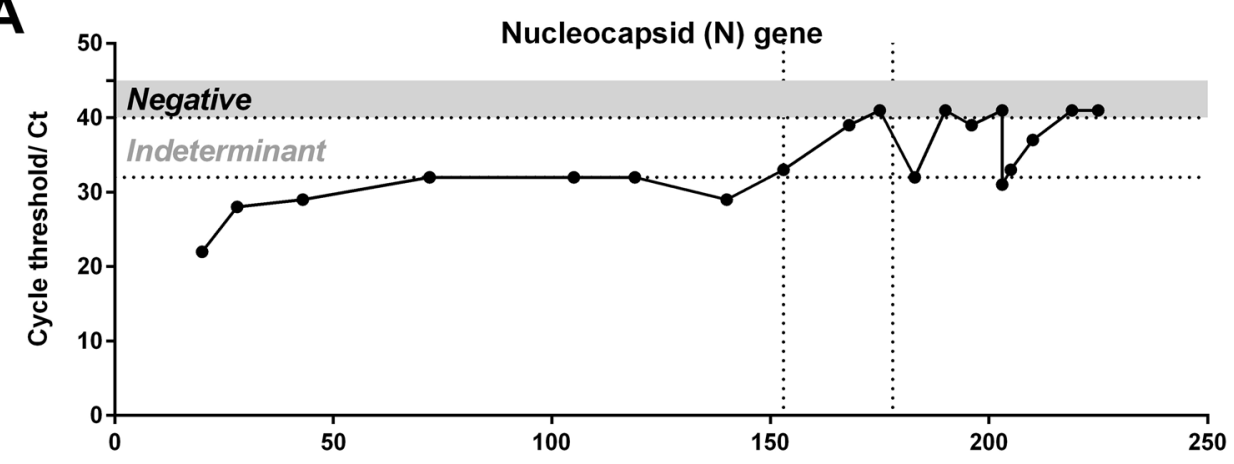

B

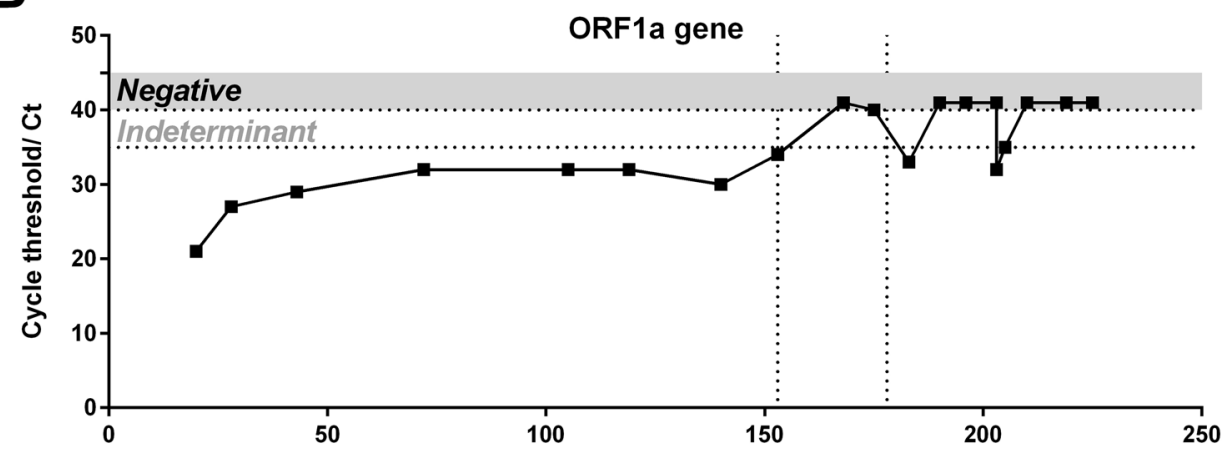

C

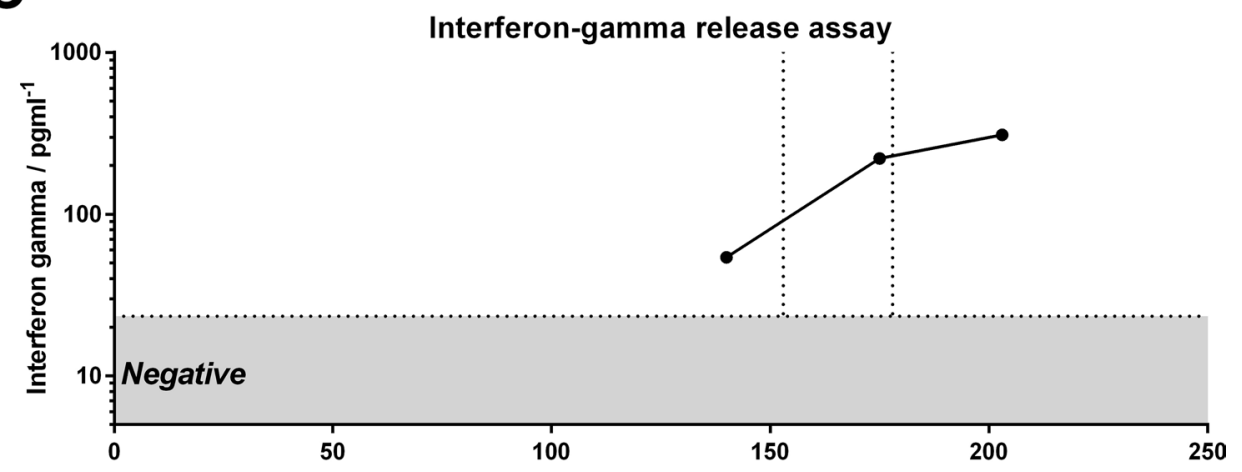

D

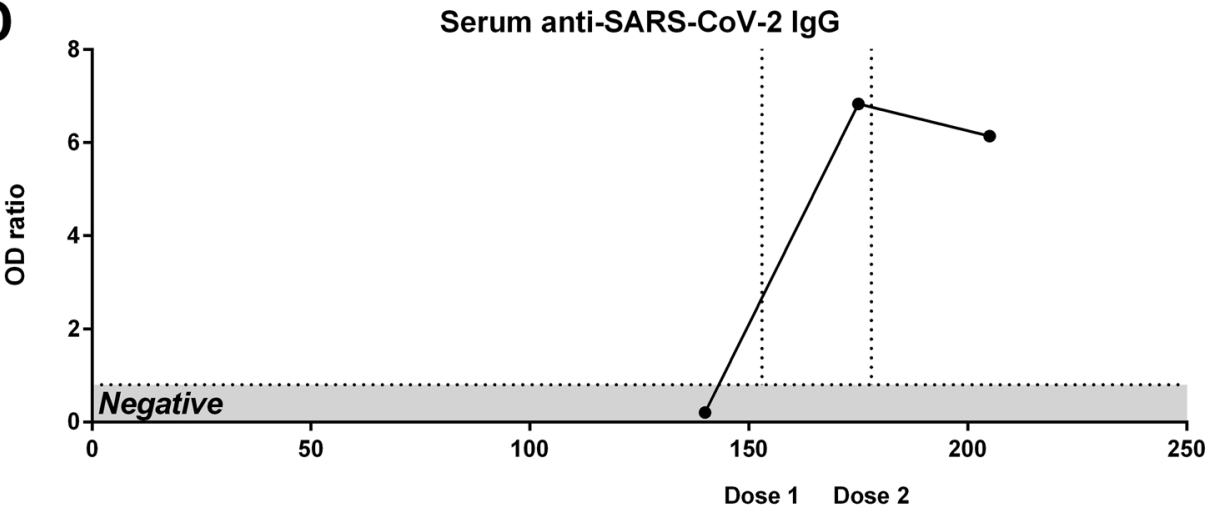

Interval following first symptom onset/ days 
computed-tomography (CT) imaging of the chest at day 153 showed small airway inflammatory changes with widespread bilateral basal tree-in-bud and centrilobular micro-nodularity, which had evolved from imaging 5 years prior (online supplementary, Figure S1). At day 140, anti-spike S1 SARSCoV-2 IgG remained absent. Whole blood-based interferongamma (IFN $\gamma$ ) release assay showed an equivocal $\mathrm{T}$ cell response to a SARS-CoV-2 peptide megapool covering the spike, nucleocapsid, and membrane glycoproteins (see online supplementary), potentially reflecting prior HKU1infection. The absence of serum neutralizing antibodies and immunocompromised status have previously been associated with isolation of infectious SARS-CoV-2 from the respiratory tract and in view of the individual's occupation requiring close contact with members of the public, self-isolation was continued.

Given persistent RT-PCR positivity, ongoing fluctuating symptoms and impact on both work and mental health of prolonged self-isolation, we adopted an individualized therapeutic approach. A growing range of therapeutic strategies have been suggested to counter SARS-CoV-2 infection in immunocompromised individuals including antibody therapies (convalescent plasma (CPT), hyperimmune IgG, and monoclonal antibodies) and antivirals. Rapid symptomatic and virological response have been reported following convalescent plasma therapy (CPT) in B cell-deficient individuals with protracted SARS-CoV-2, including where antiviral therapy with remdesivir has failed [5]. However, use of CPT has been questioned following suggestion it may accelerate vaccine-escape mutations [3]. Passive immunization with neutralizing monoclonal antibodies appear efficacious in post-exposure settings, particularly in seronegative individuals; however, this was not clinically accessible [1]. We hypothesized stimulation of the endogenous immune response through therapeutic vaccination could support viral clearance. Two doses of bnt $162 \mathrm{~b} 2 \mathrm{mRNA}$ vaccination were administered 1 month apart, with mild flu-like symptoms only. Enhanced cellular responses and seroconversion were demonstrated 14 days following the first vaccine dose (Fig. 1C, D), with anti-SARS-CoV-2 spike IgG response exceeding thresholds commonly used for selection of convalescent plasma therapy. SARS-CoV-2-specific IFN- $\gamma^{+} \mathrm{T}$ cell increased significantly following vaccination to levels comparable to those induced among healthy controls. AntiSARS-CoV-2 antibodies in immunoglobulin SCIg replacement were negative at this time (data not shown). A contemporaneous rise in RT-PCR cycle threshold $(\mathrm{Ct})$ for detection by 6 cycles was noted across both viral nucleocapsid (N)gene and ORF1a-gene targets. Given a difference of $1 \mathrm{Ct}$ unit is approximately equivalent to a factor of 2 in the number of viral particles per sample, this represents a 64-fold decrease in viral genetic material recovered by nasopharyngeal swabbing 2 weeks following initial vaccination. SARS-CoV-2 clearance followed 72 days following first therapeutic vaccination dose (day 218 following initial RT-PCR detection).

\section{Discussion}

This case highlights persistent SARS-CoV-2 infection as an important outcome in immunodeficient individuals, beyond commonly used metrics of mortality or critical illness. Persistent infection is often associated with a symptomatic burden to the individual, further amplified here by the psychological and economic impacts of prolonged isolation. Diagnosis may be reliant on a high index of clinical suspicion in order to minimize the risk of cross-infection, particularly in the hospital setting. To our knowledge, this is the first description of therapeutic mRNA vaccination in the context of persistent SARS-CoV-2 infection. Importantly, vaccination proved well-tolerated and successfully elicited humoral and cellular responses which had not been induced after 120 days of PCR-confirmed SARS-CoV-2 infection. The timing of viral clearance following induction of host immunity strongly supports a causal role for vaccination; however, we cannot exclude that viral clearance may have occurred independently. Further studies are required to assess the reproducibility and generalizability of our findings, particularly given the likely heterogeneity of vaccineinduced immune response across the spectrum of inborn errors of immunity. In conclusion, we highlight the potential for therapeutic vaccination in persistent SARS-CoV-2 infection where sufficient immunological function remains to produce relevant humoral and $\mathrm{T}$ cell responses.

Supplementary Information The online version contains supplementary material available at https://doi.org/10.1007/s10875-021-01158-5.

Acknowledgements We are grateful for the patient in allowing us to share this case. The following are members of the Immunodeficiency Centre for Wales Consortium: Kathryn Bramhall ${ }^{1}$, Colin R Price ${ }^{1}$, Kimberly Evans ${ }^{1}$, Emily Carne ${ }^{1}$, Dr Tariq El-Shanawany ${ }^{1}$, and Dr Richard Cousins ${ }^{1}$.

Author Contribution MJP and SJ conceived the study and coordinated investigations. EMC, KE, and CRP gathered clinical samples and arranged vaccination. MJS and AG performed assessment of cellular responses to SARS-CoV-2. KB performed other immunological investigations. RB and MJP collated clinical information, and wrote the first draft. All authors critically appraised and approved the final version of the manuscript.

Funding MJP is supported by the Welsh Clinical Academic Training (WCAT) program and a Career Development Award from the Association of Clinical Pathologists and is a participant in the NIH Graduate Partnership Programme. AG and MJS are supported by a UKRI COVID-19 National Core Study Immunity program ("SARS-CoV-2 Optimal Cellular Assays"), an Innovate UK SBRI award ("Assays for SARS-CoV-2 cellular immune responses"), and a program grant from 
Cancer Research Wales. AG is also supported by additional grant funding from the Wellcome Trust (grant code 209213/Z/17/Z).

\section{Declarations}

Ethical Approvals Written informed consent to participate in the COVID-19 ENLIST study (REC 20/YH/0309) was provided by the individual.

Competing Interests SJ has received support for conferences, speaker, advisory boards, trials, data and safety monitoring boards, and projects with CSL Behring, Takeda, Swedish Orphan Biovitrum, Biotest, Binding Site, Grifols, BPL, Octapharma, LFB, Pharming, GSK, Weatherden, Zarodex, Sanofi, and UCB Pharma. None of these conflicts relates to the current work. TE has received support for education, speaker, advisory boards, and/or research from Allergy Therapeutics, CSL Behring, Mylan, Novartis, Pharming, Takeda, and Thermo Fisher. MJS is a founder of and holds equity in ImmunoServ Ltd. The remaining authors have no potential relevant conflicts of interests to declare.

Open Access This article is licensed under a Creative Commons Attribution 4.0 International License, which permits use, sharing, adaptation, distribution and reproduction in any medium or format, as long as you give appropriate credit to the original author(s) and the source, provide a link to the Creative Commons licence, and indicate if changes were made. The images or other third party material in this article are included in the article's Creative Commons licence, unless indicated otherwise in a credit line to the material. If material is not included in the article's Creative Commons licence and your intended use is not permitted by statutory regulation or exceeds the permitted use, you will need to obtain permission directly from the copyright holder. To view a copy of this licence, visit http://creativecommons.org/licenses/by/4.0/.

\section{References}

1. E Moran T Cook AL Goodman RK Gupta S Jolles DK Menon 2021 Persistent SARS-CoV-2 infection: the urgent need for access to treatment and trials. Lancet Infect Dis. https://doi.org/10.1016/ S1473-3099(21)00464-3

2. Williamson MK, Hamilton F, Hutchings S, Pymont HM, Hackett M, Arnold D, et al. Chronic SARS-CoV-2 infection and viral evolution in a hypogammaglobulinaemic individual. medRxiv. 2021;2021.05.31.21257591.

3. Kemp SA, Collier DA, Datir RP, Ferreira IATM, Gayed S, Jahun A, et al. SARS-CoV-2 evolution during treatment of chronic infection. Nature. 2021;592(7853):277-82.

4. Dunn G, Klapsa D, Wilton T, Stone L, Minor PD, Martin J. Twenty-eight years of poliovirus replication in an immunodeficient individual: impact on the global polio eradication initiative. PLOS Pathogens. 2015;11(8):e1005114.

5. Buckland MS, Galloway JB, Fhogartaigh CN, Meredith L, Provine NM, Bloor S, et al. Treatment of COVID-19 with remdesivir in the absence of humoral immunity: a case report. Nat Commun. 2020;11(1).

Publisher's Note Springer Nature remains neutral with regard to jurisdictional claims in published maps and institutional affiliations. 